\title{
MATRIX ROUNDING AND RELATED PROBLEMS WITH APPLICATION TO DIGITAL HALFTONING
}

\author{
Naoki Katoh \\ Department of Architecture and Architectural Systems \\ Kyoto University, Japan \\ naoki@archi.kyoto-u.ac.jp
}

Abstract In this paper, we first study the problem of rounding a real-valued matrix into an integer-valued matrix to minimize an $L_{p}$-discrepancy measure between them. To define the $L_{p}$-discrepancy measure, we introduce a family $\mathcal{F}$ of regions (rigid submatrices) of the matrix, and consider a hypergraph defined by the family. The difficulty of the problem depends on the choice of the region family $\mathcal{F}$. We first investigate the rounding problem by using integer programming problems with convex piecewise-linear objective functions. Then, we propose "laminar family" for constructing a practical and well-solvable class of $\mathcal{F}$. Indeed, we show that the problem is solvable in polynomial time if $\mathcal{F}$ is the union of two laminar families. We shall present experimental results. We also give some nontrivial upper bounds for the $L_{p}$-discrepancy. We then foucs on the number of global roundings defined on a hypergraph $H_{G}=\left(V, \mathcal{P}_{G}\right)$ which corresponds to a set of shortest paths for a weighted graph $G=(V, E)$. For a given real assignment a on $V$ satisfying $0 \leq \mathbf{a}(v) \leq 1$, a global rounding $\alpha$ with respect to $H_{G}$ is a binary assignment satisfying that $\left|\sum_{v \in F} \mathbf{a}(v)-\alpha(v)\right|<1$ for every $F \in \mathcal{P}_{G}$. We conjecture that there are at most $|V|+1$ global roundings for $H_{G}$.

\section{Introduction}

Rounding is an important operation in numerical computation, and plays key roles in digitization of analogue data. Rounding of a real number $a$ is basically a simple problem: We round it to either $\lfloor a\rfloor$ or $\lceil a\rceil$, and we usually choose the one nearer to $a$. However, we often encounter a data consisting of more than one real numbers instead of a singleton. If it has $n$ numbers, we have $2^{n}$ choices for rounding. If the original data set has some feature, we need to choose a rounding so that the 
rounded result inherits as much of the feature as possible. The feature is described by using some combinatorial structure; we indeed consider a hypergraph $\mathcal{H}$ on the set. A typical input set is a multi-dimensional array of real numbers, and we consider a hypergraph whose hyperedges are its subarrays with contiguous indices. In this paper, we first focus on two-dimensional arrays; In other words, we consider rounding problems on matrices. We then consider the roounding problems on hypergraphs derived from a set of shortest paths of a weighted graph.

This article is a summary of our recent papers $[4,3]$.

\subsection{Rounding Problem and Discrepancy Measure}

Given an $N \times N$ matrix $A=\left(a_{i j}\right)_{1 \leq i, j \leq N}$ of real numbers, its rounding is a matrix $B=\left(b_{i j}\right)_{1 \leq i, j \leq N}$ of integral values such that $b_{i j}$ is either $\left\lfloor a_{i j}\right\rfloor$ or $\left\lceil a_{i j}\right\rceil$ for each $(i, j)$. There are $2^{N^{2}}$ possible roundings of a given $A$, and we would like to find an optimal rounding with respect to a given criterion. This is called the matrix rounding problem. Without loss of generality, we can assume that each entry of $A$ is in the closed interval $[0,1]$ and each entry is rounded to either 0 or 1 .

In order to give a criterion to determine the quality of roundings, we define a distance in the space of all $[0,1]$-valued $N \times N$ matrices. We introduce a family $\mathcal{F}$ of regions over the $N \times N$ integer grid

$$
G_{n}=\{(1,1),(1,2) \ldots,(N, N)\}=\left\{p_{1}, p_{2}, \ldots, p_{n}\right\}, \quad n=N^{2} .
$$

This means that each entry location of a matrix is denoted by a symbol $p_{i}$ and that the order $\left(p_{1}, \ldots, p_{n}\right)$ is arbitrary. Let $\mathcal{A}=\mathcal{A}\left(G_{n}\right)$ be the space of all $[0,1]$-valued matrices with the index set $G_{n}$, and let $\mathcal{B}=\mathcal{B}\left(G_{n}\right)$ be its subset consisting of all $\{0,1\}$-valued matrices. Let $R$ be a region in $\mathcal{F}^{1}$. For an element $A \in \mathcal{A}$, let $A(R)$ be the sum of entries of $A$ located in the region $R$, that is, $A(R)=\sum_{p_{i} \in R} a_{p_{i}}$. We define a distance $\operatorname{Dist}_{p}^{\mathcal{F}}\left(A, A^{\prime}\right)$ between two elements $A$ and $A^{\prime}$ in $\mathcal{A}$ for a positive integer $p$ by

$$
\operatorname{Dist}_{p}^{\mathcal{F}}\left(A, A^{\prime}\right)=\left[\sum_{R \in \mathcal{F}}\left|A(R)-A^{\prime}(R)\right|^{p}\right]^{1 / p} .
$$

The distance is called the $L_{p}$-distance with respect to $\mathcal{F}$. The $L_{\infty}$ distance with respect to $\mathcal{F}$ is defined by

$$
\operatorname{Dist}_{\infty}^{\mathcal{F}}\left(A, A^{\prime}\right)=\lim _{p \rightarrow \infty} \operatorname{Dist}_{p}^{\mathcal{F}}\left(A, A^{\prime}\right)=\max _{R \in \mathcal{F}}\left|A(R)-A^{\prime}(R)\right| .
$$

Using the notations above, we can formally define the matrix rounding problem: 


\section{$L_{p}$-Optimal Matrix Rounding Problem:}

$\mathcal{P}\left(G_{n}, \mathcal{F}, p\right)$ : Given a [0,1]-matrix $A \in \mathcal{A}$, a family $\mathcal{F}$ of subsets of $G_{n}$, and a positive integer $p$, find a $\{0,1\}$-matrix $B \in \mathcal{B}$ that minimizes

$$
\operatorname{Dist}_{p}^{\mathcal{F}}(A, B)=\left[\sum_{R \in \mathcal{F}}|A(R)-B(R)|^{p}\right]^{1 / p} .
$$

Also, we are interested in the following combinatorial problem:

\section{$L_{p}$-Discrepancy Bound:}

Given a $[0,1]$-matrix $A \in \mathcal{A}$, a family $\mathcal{F}$ of subsets of $G_{n}$, and a positive integer $p$, investigate upper and lower bounds of

$$
\mathcal{D}\left(G_{n}, \mathcal{F}, p\right)=\sup _{A \in \mathcal{A}} \min _{B \in \mathcal{B}} \operatorname{Dist}_{p}^{\mathcal{F}}(A, B) .
$$

The pair $\left(G_{n}, \mathcal{F}\right)$ defines a hypergraph on $G_{n}$, and $\mathcal{D}\left(G_{n}, \mathcal{F}, \infty\right)$ is called the inhomogeneous discrepancy of the hypergraph. Abusing the notation, we call $\mathcal{D}\left(G_{n}, \mathcal{F}, p\right)$ the (inhomogeneous) $L_{p}$-discrepancy of the hypergraph, and also often call $\operatorname{Dist} t_{p}^{\mathcal{F}}(A, B)$ the $L_{p}$-discrepancy measure of (quality of) the output $B$ with respect to $\mathcal{F}$.

\subsection{Motivation and our Application}

The most popular example of the family $\mathcal{F}$ is the set of all rectangular subregions in $G_{n}$, and the corresponding $L_{\infty}$-discrepancy measure is utilized in many application areas such as Monte Carlo simulation and computational geometry. Unfortunately, if we consider the family of all rectangular subregions, the discrepancy bound (for the $L_{\infty}$ measure) is known to be $\Omega(\log n)$ and $O\left(\log ^{3} n\right)$ [7]. It seems hard to find an optimal solution to minimize the discrepancy. In fact, it is NP-hard [2].

Therefore, we seek a family of regions for which low discrepancy rounding is useful in an important application and also can be computed in polynomial time. For the application, $L_{\infty}$ rounding is not always suitable, and $L_{p}$-discrepancy (with $p=1$ or 2 ) is preferable. For the purpose, we present a geometric structure of a family of regions reflecting the combinatorial discrepancy bound and computational difficulty of the matrix rounding problem.

In particular, we focus on the digital halftoning application of the matrix rounding problem, where we should consider smaller families of rectangular subregions as $\mathcal{F}$. More precisely, the input matrix represents a digital (gray) image, where $a_{i j}$ represents the brightness level of the $(i, j)$-pixel in the $N \times N$ pixel grid. Typically, $N$ is between 256 and 4096 , and $a_{i j}$ is an integral multiple of $1 / 256$ : This means that we use 256 brightness levels. If we want to send an image using fax or print it 
out by a dot (or ink-jet) printer, brightness levels available are limited. Instead, we replace $A$ by an integral matrix $B$ so that each pixel uses only two brightness levels. Here, it is important that $B$ looks similar to $A$; in other words, $B$ should be a good approximation of $A$.

For each pixel $(i, j)$, if the average brightness level of $B$ in each of its neighborhoods is similar to that of $A$, we can expect that $B$ is a good approximation of $A$. For this purpose, the set of all rectangles is not suitable (i.e., it is too large), and we may use a more compact family. Moreover, since human vision detects global features, the $L_{1}$ or $L_{2}$ measure should be better than the $L_{\infty}$ measure to obtain a clear output image. This intuition is supported by our experimental results.

We should mention here that there exist known algorithms for digital halftoning. We briefly review two popular methods among them. The first one is ordered Dither which extends a simple thresholding in such a way that it uses different thresholds depending on the positions [6]. Namely, we prepare an $M \times M$ matrix of integers ranging from 1 to $M^{2}$. This matrix (dither matrix) is tiled periodically to cover the whole image. Each pixel $a_{i j}$ in the image is compared with the corresponding threshold (the integer of dither matrix divided by $M^{2}$ ) to decide whether the output $b_{i j}=0$ or 1 . The second method is called error diffusion which propagates the quantization erros to unprocessed neighboring pixels according to a predeteremined way. More precisely, pixels are processed in a raster order, from left to right and from top to bottom. Each pixel is compared with a fixed threshold, 0.5 and round it up if it is greater than or equal to the threshold and round it down otherwise. The quantization error caused by the rounding is diffuesed over the pixels around it with fixed ratios. For example, if a pixel level is 0.7 , it is rounded up to 1 and the error -0.3 is diffused to the unprocessed pixels nearby.

This method gives excellent image quality in many cases, but it tends to produce visible artifacts in an area of uniform intensity.

Those two existing methods do not have any theoretical background which guarantee the quality of the goodness of the output image. This is one of the motivations of our theoretical study.

\subsection{Known Results on $L_{\infty}$ Measure}

For the $L_{\infty}$ measure, the following beautiful combinatorial result is classically known:

THEOREM 1 [Baranyai[5]1974] Given a real-valued matrix $A=\left(a_{i j}\right)$ and a family $\mathcal{F}$ of regions consisting of all rows, all columns and the whole matrix, there exists an integer-valued matrix $B=\left(b_{i j}\right)$ such that $\mid A(R)-$ $B(R) \mid<1$ holds for every $R \in \mathcal{F}$. 
The combinatorial structure and algorithmic aspects of roundings of (one-dimensional) sequences with respect to the $L_{\infty}$-discrepancy measure are investigated in recent studies $[2,17]$.

The incidence matrix $\mathcal{C}\left(G_{n}, \mathcal{F}\right)=\left(\mathcal{C}_{i j}\right)$ of the hypergraph $\left(G_{n}, \mathcal{F}\right)$ is defined by $\mathcal{C}_{i j}=1$ if the $j$-th element of $G_{n}$ belongs to the $i$-th region $R_{i}$ in $\mathcal{F}$ and 0 otherwise. ${ }^{2}$ A hypergraph is called unimodular if its incidence matrix is totally unimodular, where a matrix $\mathcal{C}$ is totally unimodular if the determinant of each square submatrix of $\mathcal{C}$ is equal to 0,1 , or -1 .

The $L_{\infty}$-discrepancy problem can be formulated as an integer programming problem, and the unimodularity implies that its relaxation has an integral solution. A classical theorem of Ghouila-Houri [9]implies that total unimodularity is a necessary and sufficient condition for the existence of a rounding with $L_{\infty}$ discrepancy less than 1 .

\subsection{Organization of the Paper}

We shall consider $L_{p}$-discrepancy measure instead of $L_{\infty}$-discrepancy measure. Starting with one-dimensional array, we shall show several interesting unimodular families of $\mathcal{F}$ where we can find an optimal solution of $\mathcal{P}\left(G_{n}, \mathcal{F}, p\right)$. we consider the optimization problem. In fact, if the hypergraph is unimodular, the rounding minimizing the $L_{p}$-discrepancy can be computed in polynomial time by translating it to a separable convex programming problem and applying known general algorithms [10]. However, we want to define a class of region families for which we can compute the optimal solution more efficiently, as well as the class is useful in applications (in particular, the digital halftoning application). We consider the union of two laminar families (defined in Section 3), and show that the matrix rounding problem can be formulated into a minimum cost flow problem, and hence solved in polynomial time. We then show recent results on the $L_{p}$-discrepancy bound [4].

We implemented the algorithm using LEDA[11]. Some output pictures of the algorithm applying to the digital halftoning problem are included.

Finally, we briefly review the results concerning global roundings.

\section{Mathematical Programming Formulations}

\subsection{One-Dimensional Case}

We shall begin with a basic case for the rounding problem. We take a $[0,1]$-valued one-dimensional array $A=\left(a_{i}\right)_{i=1,2, \ldots, n}$ as an input, and a binary array $B=\left(b_{i}\right)_{i=1,2, \ldots, n}$ as an output. A family $\mathcal{F}$ of regions we consdier here is a set of all subintervals of the entire interval $[1, n]$. Now 
the $L_{1}$-optimal rounding problem on $\mathcal{F}$ is described as follows:

$$
(I 1): \text { minimize }\left\{\sum_{I_{i} \in \mathcal{F}}\left|\sum_{j \in I_{i}} a_{j}-\sum_{j \in I_{i}} b_{j}\right| \mid b_{j}=0,1, \quad j=1,2, \ldots, n\right\} .
$$

If we introduce new variables $y_{i}=B\left(I_{i}\right)$ and constants $c_{i}=A\left(I_{i}\right)$ the problem can be replaced by

$$
\begin{array}{ll}
(I 2): \quad \operatorname{minimize} & \sum_{I_{i} \in \mathcal{F}}\left|y_{i}-c_{i}\right| \\
\text { subject to } & y_{i}=\sum_{j \in I_{i}} b_{j}, j=1,2, \ldots, m=|\mathcal{F}| \text { and } \\
& b_{j}=0,1, \quad j=1,2, \ldots, n .
\end{array}
$$

The constarints concerning the variables $y_{i}$ are represented in a form:

$$
\left(-I, \mathcal{C}\left(G_{n}, \mathcal{F}\right)\right) Y=0
$$

where $I$ is an identity matrix and $Y=\left(y_{1}, \ldots, y_{m}, b_{1}, \ldots, b_{n}\right), G_{n}=$ $\{1,2, \ldots, n\}$ and $\mathcal{C}\left(G_{n}, \mathcal{F}\right)$ is the incidence matrix defined by $c_{i j}=$ 1 if $b_{j} \in I_{i}, 0$, otherwise. Notice that the above problem can be viewed as a separable piecewise linear convex minimization problem under linear constraints. It is known that the incidence matrix $\mathcal{C}\left(G_{n}, \mathcal{F}\right)$ is totally unimodular [18]. Thus, using the following theorem, the problem (I2) can be solve in polynomial time.

Theorem 2 [Hochbaum and Shanthikumar [10]] A nonlinear separable convex optimization problem $\min \left\{\sum_{i=1}^{n} f_{i}\left(x_{i}\right) \mid A x \geq b\right\}$ on linear constraints with a totally unimodular matrix $A$ can be solved in polynomial time in $n$.

\subsection{Mathematical Programming Formulation of Matrix Rounding Problem}

We will extend the formulation of the one-dimensional rounding problem to that for matrix rounding problems.

Introducing a new variable $y_{i}=B\left(R_{i}\right)=\sum_{(j, k) \in R_{i}} b_{j k}$ for each $R_{i} \in$ $\mathcal{F}$, the problem $\mathcal{P}\left(G_{n}, \mathcal{F}, p\right)$ is described in the following form:

$$
\begin{aligned}
&(P 1): \quad \text { minimize } {\left[\sum_{R_{i} \in \mathcal{F}}\left|y_{i}-A\left(R_{i}\right)\right|^{p}\right]^{1 / p} } \\
& \text { subject to } \quad y_{i}=\sum_{(j, k) \in R_{i}} b_{j k}, i=1, \ldots, m=|\mathcal{F}| \\
& B \in \mathcal{B}\left(G_{n}\right) .
\end{aligned}
$$


Notice that $G_{n}$ denotes the one of (1) from now one. The objective function can be replaced with $\sum_{R_{i} \in \mathcal{F}}\left|y_{i}-c_{i}\right|^{p}$, where $c_{i}=A\left(R_{i}\right)=$ $\sum_{(j, k) \in R_{i}} a_{j k}$ is a constant depending only on input values. Now, $\left|y_{i}-c_{i}\right|^{p}$ is a convex function independent of other $y_{j}$ s. The constraints $y_{i}=$ $\sum_{(j, k) \in R_{i}} b_{j k}, i=1, \ldots, m$ are represented by $\left(-I, \mathcal{C}\left(G_{n}, \mathcal{F}\right)\right) Y=0$ using the incidence matrix $\mathcal{C}\left(G_{n}, \mathcal{F}\right)$ defined in Section 1.3 where $Y=$ $\left(y_{1}, \ldots, y_{m}, b_{11}, \ldots, b_{n n}\right)^{T}$ and $I$ is an identity matrix.

Although the objective function is now a separable convex function, its nonlinearity gives difficulty to analyze the properties of the solution. Thus, we apply the idea of Hochbaum and Shanthikumar [10] to replace $\left|y_{i}-c_{i}\right|^{p}$ with a piecewise linear convex continuous function $f_{i}\left(y_{i}\right)$ which is equal to $\left|y_{i}-c_{i}\right|^{p}$ for each integral value of $y_{i}$ in $\left[0,\left|R_{i}\right|\right]$. This is because we only need integral solutions, and if each $b_{p_{j}}$ is integral, $y_{i}$ must be a nonnegative integer less than or equal to $\left|R_{i}\right|$. Typically for $p=1, f_{i}\left(y_{i}\right)$ is illustrated in Figure 1.

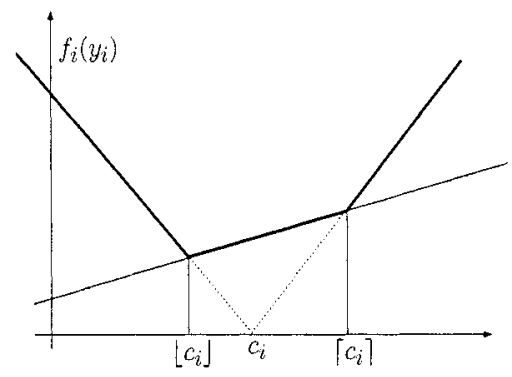

Figure 1. Conversion of the convex objective function $\left|y_{i}-c_{i}\right|$ into a piecewise linear convex function $f_{i}\left(y_{i}\right)$ with integral breakpoints (shown in bold lines).

Thus, we obtain the following problem (P2):

$(P 2): \operatorname{minimize}\left\{\sum_{R_{i} \in \mathcal{F}} f_{i}\left(y_{i}\right) \mid y_{i}=\sum_{(j, k) \in R_{i}} b_{j k}, i=1, \ldots, m, B \in \mathcal{B}\left(G_{n}\right)\right\}$

Here $m=|\mathcal{F}|$. Thus, we can formulate the problem into an integer programming problem where the objective function is a separable piecewiselinear convex function.

Let (P3) be the continuous relaxation obtained from (P2) by replacing the integral condition of $b_{i j}$ with the condition $0 \leq b_{i j} \leq 1$. Note that this is different from the continuous relaxation of $(\mathrm{P} 1)$, since the objective function of (P2) is larger than that of (P1) at non-integral values. If the matrix is totally unimodular, (P3) has an integral optimal solution by Theorem 2. This is a key to derive discrepancy bounds and also algorithms. We thus have the following result. 
COROLlary 3 The matrix rounding problem $\mathcal{P}\left(G_{n}, \mathcal{F}, p\right)$ is solved in polynomial time in $n$ if its associated incidence matrix $\mathcal{C}\left(G_{n}, \mathcal{F}\right)$ is totally unimodular.

\section{Geometric Families of Regions Defining Unimodular Hypergraphs}

In this section we consider interesting classes of families whose associated incidence matrices are totally unimodular. We call such a family a unimodular family, since the associated hypergraph is unimodular. A family $\mathcal{F}=\left\{R_{1}, R_{2}, \ldots, R_{m}\right\}$ is a partition family (or a partition) of $G_{n}$ if $\bigcup_{i=1}^{m} R_{i}=G_{n}$ and $R_{i} \cap R_{j}=\emptyset$ for any $R_{i} \neq R_{j}$ in $\mathcal{F}$. A $k$-partition family is a family of regions on a matrix which is the union of $k$ different partitions of $G_{n}$.

A family $\mathcal{F}$ of regions on a grid $G_{n}$ is a laminar family if one of the following holds for any pair $R_{i}$ and $R_{j}$ in $\mathcal{F}$ : (1) $R_{i} \cap R_{j}=\emptyset$, (2) $R_{i} \subset R_{j}$ and (3) $R_{j} \subset R_{i}$. The family is also called a laminar decomposition of the grid $G_{n}$. In general, a k-laminar family is a family of regions on a matrix which is the union of $k$ different laminar families.

The following theorem is known [18].

THEOREM 4 A 2-laminar family is unimodular.

Direct applications of Theorem 4 lead to various unimodular families of regions. The family of regions defined in Baranyai's theorem is a 2laminar family. Also, take any 2-partition family consisting of $2 \times 2$ regions on a matrix. For example, take all $2 \times 2$ regions with their upper left corners located in even points (where the sums of their row and column indices are even). The set of all those regions defines two partition families $\mathcal{F}_{\text {even }}$ and $\mathcal{F}_{\text {odd }}$ where $\mathcal{F}_{\text {even }}$ (resp. $\mathcal{F}_{\text {odd }}$ ) consists of all $2 \times 2$ squares with their upper left corners lying at even (resp. odd) rows (see Figure 2). This kind of families plays an important role in the following Section and also in our experiment. Notice that a 3 -partition family is not unimodular in general.
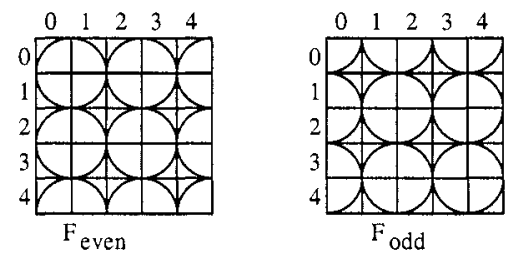

Figure 2. 2-partition family of $2 \times 2$ regions. 


\section{Algorithms for Computing the Optimal Rounding}

The arguments so far guarantee polynomial-time solvability of our problem. However, we needed a more practical algorithm for our experiments that runs fast for large-scale problem instances. In this section we will show how to solve the matrix rounding problem for a 2-laminar family based on the minimum-cost flow algorithm.

Our main result is the following:

TheOREM 5 Given a $[0,1]$-matrix $A$ and a 2-laminar family $\mathcal{F}$, an optimal binary matrix $B$ that minimizes the distance $\operatorname{Dist}_{p}^{\mathcal{F}}(A, B)$ is computed in $O\left(n^{2} \log ^{2} n\right)$ time, where $n$ is the number of matrix elements.

Proof. We can transform the problem into that of finding a minimumcost circulation flow in the network defined as follows.

Let $\mathcal{F}$ be a 2-laminar family given as the union of two laminar families $\mathcal{F}_{1}=\left\{R_{0}, R_{1}, \ldots, R_{m}\right\}$ and $\mathcal{F}_{2}=\left\{R_{0}^{\prime}, R_{1}^{\prime}, \ldots, R_{m^{\prime}}^{\prime}\right\}$ over the grid $G_{n}$, where $R_{0}$ and $R_{0}^{\prime}$ are the entire region $G_{n}$. The network to be constructed consists of three parts. The first part is an in-tree $T_{1}$ derived from $\mathcal{F}_{1}$ whose root is $R_{0}$, the second one an out-tree $T_{2}$ from $\mathcal{F}_{2}$ whose root is $R_{0}^{\prime}$, and the third part connects $T_{1}$ and $T_{2}$. The lattice structure implied by $\mathcal{F}_{1}$ naturally defines an in-tree $T_{1}$ such that the vertex set is the set of regions in $\mathcal{F}_{1}$ and there is a directed edge $\left(R_{i}, R_{j}\right)$ if and only if $R_{i} \subseteq R_{j}$ and there is no other region $R_{k}$ such that $R_{j} \subseteq R_{k} \subseteq R_{i}$. Then, each region $R_{i}, i \geq 1$ has a unique outgoing edge, which is denoted by $e\left(R_{i}\right)$. We can similarly define $T_{2}$ for the laminar family $\mathcal{F}_{2}$, in which the edge direction is reversed in $T_{2}$, that is, each node $R_{i}^{\prime}, i \geq 1$ has an unique incoming edge, which is denoted by $e\left(R_{i}^{\prime}\right)$.

In addition, leaves of $T_{1}$ and $T_{2}$ are connected by edges corresponding to elements of $G_{n}$. Because of the definition of $\mathcal{F}_{1}$ and $\mathcal{F}_{2}$, each element $(k, l)$ of $G_{n}$ belongs to exactly one region in $\mathcal{F}_{1}$ which is a leaf in $T_{1}$ and to exactly one region in $\mathcal{F}_{2}$ which is a leaf in $T_{2}$. If $(k, l)$ belongs to $R_{i}$ and $R_{j}^{\prime}$, then we have a directed edge $e(i, j)$ from $R_{j}^{\prime}$ to $R_{i}$. Finally, we draw an edge from $R_{0}$ to $R_{0}^{\prime}$.

Now, we define capacity and cost coefficient of each edge. (The lower bound on the flow of each edge is defined to be 0 .) The capacity of an edge $e(i, j)$ is determined simply as 1 because $b_{i j}$ is to be rounded to 0 or 1 . Other edges do not have capacity constraint.

The cost associated of an edge $e\left(R_{i}\right)\left(e\left(R_{j}^{\prime}\right)\right.$, respectively) is $f_{i}\left(y_{i}\right)$ where $y_{i}$ is defined in $(P 1)$. As already mentioned, $f_{i}\left(y_{i}\right)$ is piecewise linear convex in $y_{i}$. We then apply the result of $[16]$ (see also Chapter 
14 of the book by Ahuja et al. [1]) for the algorithm of minimum-cost circulation with separable convex costs.

From this result, we can find an optimal rounding in time $O(|E| \log$ $U(|E|+|V| \log |V|))$ for a network with node set $V$ and edge set $E$ and the largest integral capacity $U$. In our case, $|V|,|E|$ and $U$ are all $O(n)$, and thus we have $O\left(n^{2} \log ^{2} n\right)$, where $n$ is the number of matrix elements. Q.E.D.

\section{Upper Bounds for the $L_{p}$-Discrepancy}

In this section, we show the following theorem for the $L_{p}$-discrepancy of a unimodular family. We will omit the proof for the space limit.

Theorem 6 [Asano, Katoh, Obokata and Tokuyama [4]] If $\mathcal{F}$ is unimodular and $p \leq 3$, for any $A \in \mathcal{A}$ we have

$$
\min _{B \in \mathcal{B}} \operatorname{Dist}_{p}^{\mathcal{F}}(A, B) \leq \frac{1}{2}|\mathcal{F}|^{1 / p} .
$$

It is easy to give an instance to show that the bound is tight: Consider Baranyai's problem on a matrix having $\frac{1}{2}$ entries in its diagonal position (other entries are zeros).

\section{Application to Digital Halftoning}

The quality of color printers has been drastically improved in recent years, mainly based on the development of fine control mechanism. On the other hand, there seems to be no great invention on the software side of the printing technology. What is required is a technique to convert a continuous-tone image into a binary image consisting of black and white dots so that the binary image looks very similar to the input image. From a theoretical standpoint, the problem is how to approximate an input $[0,1]$-array by a binary array. Since this is one of the central techniques in computer vision and computer graphics, a great number of algorithms have been proposed (see, e.g., $[12,8,6,13,15]$ ). However, there have been very few studies toward the goal of achieving an optimal binary image under some reasonable criterion; maybe because the problem itself is very practically oriented. A desired output image is the one which looks similar to the input image to the human visual system. The most popular distortion criterion that is used in practice is perhaps Frequency Weighted Mean Square Error (FWMSE)[14]which is defined by

$$
W(G, X)=\sum_{(i, j) \in G_{n}}\left[\sum_{k=-K}^{K} \sum_{l=-K}^{K} v_{|k||l|} a_{i+k, j+l}-\sum_{k=-K}^{K} \sum_{l=-K}^{K} v_{|k||l|} b_{i+k, j+l}\right]^{2} .
$$


Here, $V=\left(v_{|k| l|l|}\right), k, l=0, \ldots, K$ is an impulse response that approximates the characteristics of the human visual system and $K$ is some small constant, say 3. Our discrepancy measure which has been discussed in this paper is a hopeful replacement; Indeed, the $L_{2}$-discrepancy measure can be regarded as a simplified version of the FWMSE criterion.

We have implemented the algorithm using LEDA [11]functions for finding minimum-cost flow, and applied to several test images to compare its results with the error diffusion algorithm which is most commonly used in practice. The data we used for our experiments are Standard high precision picture data created by the Institute of Image Electronics Engineers of Japan, which include four standard pictures called, "Bride," "Harbor," "Wool," and "Bottles." They are color pictures of 8 bits each in RGB. Their original picture size is $4096 \times 3072$. In our experiments we scaled them down to $1024 \times 768$ in order to shorten the running time of the program. Figures 3 and 4 show experimental results for "Wool" and "Wine" to compare our algorithm with error diffusion. Our algorithm has been implemented using a 2-laminar family defined by the two tiles (b) and (c) depicted in Figure 5.
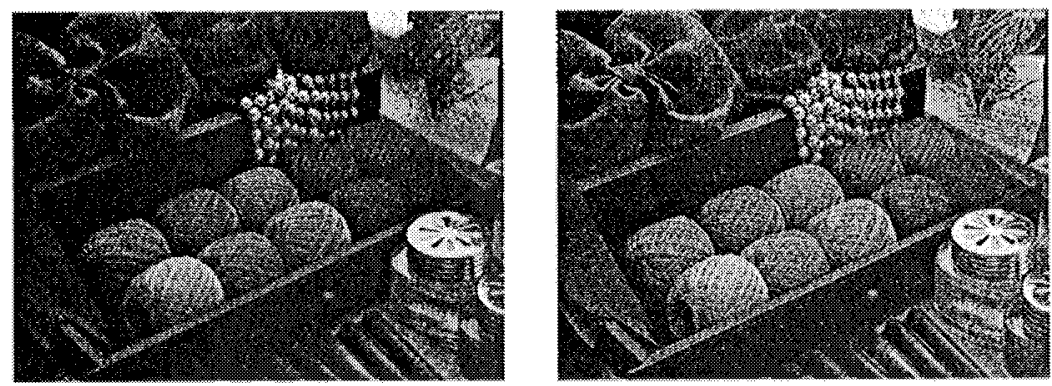

Figure 3. Experimental results for "Wool". Output images by error diffusion algorithm (left) and the algorithm in this paper (right).

\section{Global Roundings}

Let $H=(V, \mathcal{F})$, where $\mathcal{F} \subset 2^{V}$, be a hypergraph on a set $V$ of $n$ nodes. Given a real valued function a on $V$, we say that an integer valued function $\alpha$ on $V$ is a global rounding of a with respect to $H$, if $w_{F}(\alpha)$ is a rounding of $w_{F}(\mathbf{a})$ for each $F \in \mathcal{F}$, where $w_{F}(f)$ denotes $\sum_{v \in F} f(v)$. Without loss of generality we restrict our attention to the case where the ranges of $\mathbf{a}$ and $\alpha$ are $[0,1]$ and $\{0,1\}$ respectively.

This notion of global roundings on hypergraphs is closely related to

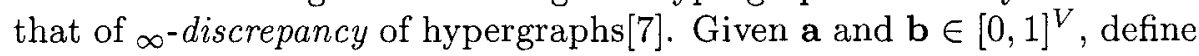



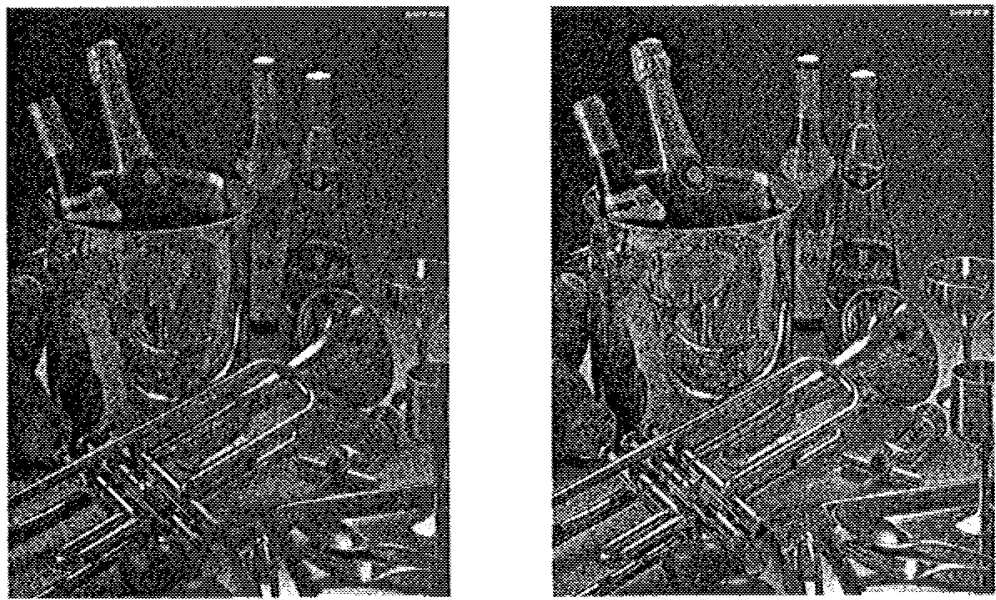

Figure 4. Experimental results for "Wine". Output images by error diffusion (left) and by the algorithm in this paper (right).

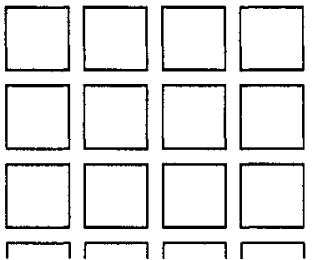

(a)

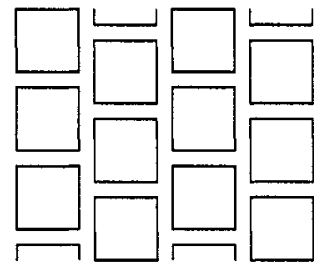

(b)

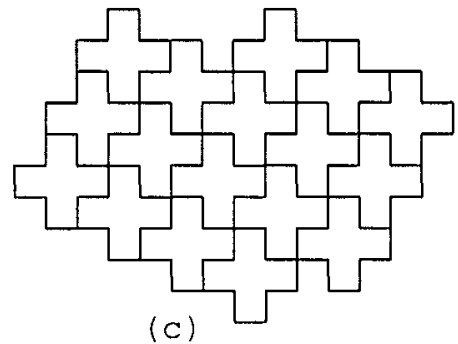

(c)

Figure 5. Three different partitions of the image plane (a) by $2 \times 2$ squares, (b) vertically shifted $2 \times 2$ squares, and (3) cross patterns consisting of 5 pixels.

the discrepancy $D_{H}(\mathbf{a}, \mathbf{b})$ between them on $H$ by

$$
D_{H}(\mathbf{a}, \mathbf{b})=\max _{F \in \mathcal{F}}\left|w_{F}(\mathbf{a})-w_{F}(\mathbf{b})\right| .
$$

Our recent paper [3]investigated maximum number $\nu(H)$ of global roundings.

This direction of research is initiated by Sadakane et al.[17] where the authors discovered a somewhat surprising fact that $\nu\left(I_{n}\right) \leq n+1$ where $I_{n}$ is a hypergraph on $V=\{1,2, . ., n\}$ with edge set $\{[i, j] ; 1 \leq i \leq j \leq n\}$ consisting of all subintervals of $V$. We can also see that $\nu(H) \geq n+1$ for any hypergraph $H$ : if we let $\mathbf{a}(v)=\epsilon$ for every $v$, where $\epsilon<1 / n$, then any binary assignment on $V$ that assigns 1 to at most one vertex is a global rounding of $H$, and hence $\nu(H) \geq n+1$. 
Given this discovery, it is natural to ask for which class of hypergraphs this property $\nu(H)=n+1$ holds.

We showed [3] that $\nu(H)=n+1$ holds for a considerably wider class of hypergraphs. Given a connected $G$ in which edges are possibly weighted by a positive value, we define a shortest-path hypergraph $H_{G}$ generated by $G$ as follows: a set $F$ of vertices of $G$ is an edge of $H_{G}$, if and only if $F$ is the set of vertices of some shortest path in $G$ with respect to the given edge weights. Note that we permit more than one shortest path between a pair of nodes if they have the same length. The following theorem is our main result [3]:

THEOREM $7 \nu\left(H_{G}\right)=n+1$ holds for the shortest-path hypergraph $H_{G}$, if $G$ is a tree, a cycle, a tree of cycles, an unweighted mesh, or an unweighted $k$-tree.

We conjecture that the result holds for general connected graphs.

\section{Concluding Remarks}

We have considered the matrix rounding problem based on $L_{p}$-discrepancy measure. Although we have shown that the measure is useful in application to the digital halftoning application, the current algorithm is too slow if we want to require speed together with the high-quality requirement. It is desired to design a faster algorithm (even an approximation algorithm). Moreover, it is an interesting question to investigate what kind of region families give the best criterion for the halftoning application. Once we know such a region family, it is valuable to design an algorithm (heuristic algorithm if the problem for solving the optimal solution is intractable) for the criterion.

\section{Acknowledgment}

The authors would like to thank Tetsuo Asano, Koji, Obokata, Tomomi Matsui, Koji Nakano, Hiroshi Nagamochi, and Takeshi Tokuyama for their valuable comments and helpful discussions.

\section{Notes}

1. Strictly speaking, $R$ can be any subset of $G_{n}$. Although we implicitly assume that $R$ forms some connected portion on the grid $G_{n}$, the connectivity assumption is not used throughout the paper.

2. We implicitly assume a one-dimensional ordering of elements in $G_{n}$ 


\section{References}

[1] R. Ahuja, T. Magnanti, and J. Orlin. Network Flows, Theory Algorithms and Applications. Prentice Hall, 1993.

[2] T. Asano, T. Matsui, and T. Tokuyama. Optimal roundings of sequences and matrices. Nordic Journal of Computing, 7:241-256, 2000.

[3] Tetsuo Asano, Naoki Katoh, Hisao Tamaki, and Takeshi Tokuyama. The structure and number of global roundings of a graph. Proc. of COCOON 2003, LNCS 2697, pages $130-138,2003$.

[4] Tetsuo Asano, Naoki Katoha, Koji Obokata, and Takeshi Tokuyama. Matrix rounding under the lp-discrepancy measure and its application to digital halftoning. SODA 2002 (also to appear in SIAM J. Comput.), pages 896-904, 2002.

[5] Z. Baranyai. On the factorization of the complete uniform hypergraphs. Infinite and Finite Sets, (A. Hanaj, R. Rado and V. T. Sós, eds.), Colloq. Math. Soc. J'anos Bolyai, 10:91-108, 1974.

[6] B. E. Bayer. An optimum method for two-level rendition of continuous-tone pictures. Conference Record, IEEE International Conf. on Communications, 1:26-11-26-15, 1973.

[7] J. Beck and V. T. Sós. Discrepancy Theory, in Handbook of Combinatorics, volume II. Elsevier, 1995.

[8] R. W. Floyd and L. Steinberg. An adaptive algorithm for spatial gray scale. SID 75 Digest, Society for Information Display, pages 36-37, 1975.

[9] A. Ghoulia-Houri. Characterisation des matrices totalement unimodulaires. C.R. Acad/Sci. Paris, 254:1192-1194, 1962.

[10] D.S. Hochbaum and J.G. Shanthikumar. Nonlinear separable optimization is not much harder than linear optimization. Journal of ACM, 37:843-862, 1990.

[11] http://www.algorithmic-solutions.com/as_html/products/products.html. LEDA homepage. Algorithmic Solutions Software GmbH, 2003.

[12] D.E. Knuth. D.e. knuth. ACM Trans. Graphics, 6:245-273, 1987.

[13] J. O. Limb. Design of dither waveforms for quantized visual signals. Bell Syst. Tech. J., 48-7:2555-2582, 1969.

[14] Q. Lin. Halftone image quality analysis based on a human vision model. Proceedings of SPIE, 1913:378-389, 1993.

[15] B. Lippel and M. Kurland. The effect of dither on luminance quantization of pictures. IEEE Trans. Commun. Tech., COM-19:879-888, 1971.

[16] M. Minoux. Solving integer minimum cost flows with separable cost objective polynomially. Mathematical Programming Study, 46:237-239, 1986.

[17] K. Sadakane, N. Takki-Chebihi, and T. Tokuyama. Combinatorics and algorithms on low-discrepancy roundings of a real sequence. Proc. 28th ICALP, Springer LNCS 2076, pages 166-177, 2001.

[18] A. Schrijver. Theory of Linear and Integer Programming. Wiley-Interscience Series in Discrete Mathematics. John Wiley and Sons, 1986. 